\title{
A CHARACTERIZATION OF LOCALLY FINITE VARIETIES THAT SATISFY A NONTRIVIAL CONGRUENCE IDENTITY
}

\author{
KEITH A. KEARNES
}

\begin{abstract}
We show that a locally finite variety satisfies a nontrivial congruence identity if and only if it satisfies an idempotent Mal'tsev condition that fails in the variety of semilattices.
\end{abstract}

\section{INTRODUCTION}

In [1], R. Freese and J. B. Nation prove that the variety of semilattices satisfies no nontrivial lattice identity as a congruence identity. Together with results in [6] or [7] this implies that if a variety satisfies some nontrivial congruence identity, then it satisfies an idempotent Mal'tsev condition that fails in the variety of semilattices. In this paper the converse statement is shown to hold for locally finite varieties, thereby solving Problem 13 of [2]. The machinery for the proof is developed in Lemmas 3.2 and 3.4 using tame congruence theory. Modulo these two lemmas the proof of our main result, Theorem 3.7, can be read with no knowledge of the theory.

\section{A Nice Lattice}

The following lattice plays an important role in this paper.

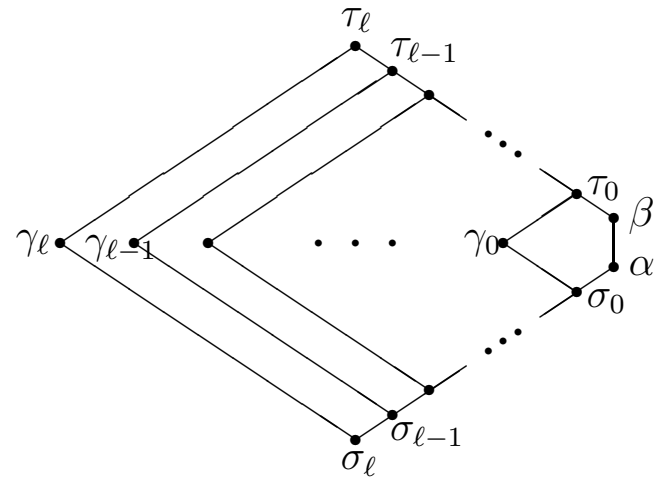

1991 Mathematics Subject Classification. Primary 08B05, Secondary 08B10.

This material is based upon work supported by the National Science Foundation under Grant No. DMS 9802922. 
We denote it by $\mathbf{N}_{\ell}$. When $\ell=0$ this lattice is a five-element nonmodular lattice that is often called the pentagon. Our notation for the pentagon, $\mathbf{N}_{0}$, is in conflict with the usual notation for it, but is more convenient for the calculations we need to do later. (Usually the pentagon is denoted $\mathbf{N}_{5}$.)

It is easy to see that $\mathbf{N}_{\ell}$ is subdirectly irreducible, and it is shown in Example 5.2 and Remark 6.3 of [5] that $\mathbf{N}_{\ell}$ is projective. Let $\mathcal{K}_{\ell}$ denote the class of all lattices which have no sublattice isomorphic to $\mathbf{N}_{\ell}$. It is immediate from the definition that $\mathcal{K}_{\ell}$ is closed under the formation of sublattices, while the subdirect irreducibility of $\mathbf{N}_{\ell}$ implies that $\mathcal{K}_{\ell}$ is closed under the formation of products, and the projectivity of $\mathbf{N}_{\ell}$ implies that $\mathcal{K}_{\ell}$ is closed under the formation of homomorphic images. Hence $\mathcal{K}_{\ell}$ is a variety. Fix an identity $\nu_{\ell}$ that holds in $\mathcal{K}_{\ell}$ but fails in $\mathbf{N}_{\ell}$. Necessarily $\nu_{\ell}$ axiomatizes $\mathcal{K}_{\ell}$ relative to the variety of all lattices, so for any lattice $\mathbf{L}$ exactly one of the following is true: $\mathbf{L} \models \nu_{\ell}$ or else $\mathbf{L}$ has a sublattice isomorphic to $\mathbf{N}_{\ell}$.

Theorem 2.1. Let $\mathcal{V}$ be a variety of algebras. $\mathcal{V}$ satisfies $\nu_{\ell}$ as a congruence identity if and only if $\mathbf{N}_{\ell}$ is not embeddable in $\operatorname{Con}(\mathbf{A})$ for any finitely generated $\mathbf{A} \in \mathcal{V}$.

Proof. It is implicit in [7] (and not hard to prove) that a variety satisfies a congruence identity if and only if its finitely generated members satisfy it. Hence, this theorem is a consequence of the remarks above.

\section{The Main Result}

We remind the reader that if $\mathbf{A}$ is a finite algebra and $\delta$ and $\theta$ are congruences of $\mathbf{A}$ for which $\delta \prec \theta$ in $\operatorname{Con}(\mathbf{A})$, then a two-element set $\{0,1\}$ is a $\langle\delta, \theta\rangle$-subtrace if $(0,1) \in \theta-\delta$ and $\{0,1\}$ is a subset of a $\langle\delta, \theta\rangle$-minimal set. Our immediate goal is to investigate how the shape of a congruence lattice influences the equations that hold on subtraces.

In this section the notation $a \equiv_{\theta} b$ means $(a, b) \in \theta$, and is read " $a$ is equivalent to $b$ modulo $\theta$ ".

Definition 3.1. Let $\mathbf{A}$ be a finite algebra with congruences $\delta \prec \theta$, and let $K=\operatorname{Int}[\delta, \theta]$ be the two-element interval in $\operatorname{Con}(\mathbf{A})$ determined by these congruences. If $f$ and $g$ are terms of $\mathbf{A}$, then we will use the notation $f\left(x_{1}, \ldots, x_{n}\right) \approx_{K} g\left(x_{1}, \ldots, x_{n}\right)$ to mean that whenever $\{0,1\}$ is a $\langle\delta, \theta\rangle$-subtrace, then $f\left(x_{1}, \ldots, x_{n}\right) \equiv_{\delta} g\left(x_{1}, \ldots, x_{n}\right)$ holds provided all $x_{i} \in\{0,1\}$.

If $\alpha<\beta$ are congruences on $\mathbf{A}$ and $I=\operatorname{Int}[\alpha, \beta]$, then we will write $f\left(x_{1}, \ldots, x_{n}\right) \approx_{I} g\left(x_{1}, \ldots, x_{n}\right)$ to mean that $f\left(x_{1}, \ldots, x_{n}\right) \approx_{K}$ 
$g\left(x_{1}, \ldots, x_{n}\right)$ holds whenever $K$ is a two-element subinterval of $I$. We call equations of the type $f \approx_{I} g$ local equations.

Lemma 3.2. Assume that $\mathbf{A}$ is a finite algebra and that $\mathrm{Con}(\mathbf{A})$ has a sublattice isomorphic to the pentagon, with congruences labeled as in the following figure.

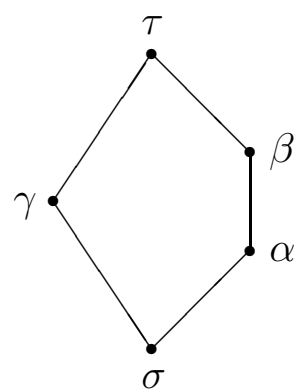

Let $I=\operatorname{Int}[\sigma, \tau]$ and let $J=\operatorname{Int}[\alpha, \beta]$. If $\mathbf{A}$ satisfies the local equation $f(x, y, y) \approx_{I} x$, then $\mathbf{A}$ satisfies both of the local equations

$$
f(x, x, y) \approx_{J} x \text { and } f(x, y, x) \approx_{J} x .
$$

Proof. It is enough to prove that if the local equation $f(x, y, y) \approx_{I} x$ holds, then the local equation $f(x, x, y) \approx_{J} x$ holds. The same argument applied to $f^{\prime}(x, y, z)=f(x, z, y)$ shows that if the local equation $f(x, y, y) \approx_{I} x$ holds, then $f(x, y, x) \approx_{J} x$ holds.

Assume to the contrary that $f(x, y, y) \approx_{I} x$ while $f(x, x, y) \not \sim_{J}$ $x$. Since $f(x, x, y) \nsim_{J} x$, there exist a two-element subinterval $K=$ Int $[\delta, \theta]$ of $J$, and a $\langle\delta, \theta\rangle$-subtrace $\{0,1\}$ such that $w=f(0,0,1) \not \equiv \delta_{\delta} 0$. We shall derive a contradiction to this.

The local equation $f(x, y, y) \approx_{I} x$ implies that

$$
f(0,1,1) \equiv_{\delta} 0 \equiv_{\delta} f(0,0,0) .
$$

Assume that typ $(\delta, \theta)=\mathbf{1}$. Then $\theta$ is strongly abelian over $\delta$ (Definition 3.9 and Theorem 5.6 of [2]), so we can replace the argument in the second position on each side of this equality with 0 and still maintain $\mathrm{a} \equiv{ }_{\delta}$-relation. This yields

$$
w=f(0,0,1) \equiv_{\delta} f(0,0,0) \equiv_{\delta} 0,
$$

which is false. Hence typ $(\delta, \theta) \neq \mathbf{1}$.

Assume now that typ $(\delta, \theta) \in\{\mathbf{4}, \mathbf{5}\}$. The $\theta$-class containing 0 and 1 is connected by a $\delta$-closed preorder that is compatible with all operations of $\mathbf{A}$, which has the property that distinct elements of a twoelement subtrace are comparable. (See Theorem 5.26 of [2]). So, if $\{x, y\}=\{0,1\}$, then $f(x, x, x)$ and $f(x, y, y)$ are comparable elements of this $\theta$-class. The element $f(x, x, y)$ is in the interior of the interval determined by $f(x, x, x)$ and $f(x, y, y)$. For us, $f(x, x, x) \equiv_{\delta} x \equiv_{\delta}$ 
$f(x, y, y)$, so we deduce that $f(x, x, y) \equiv_{\delta} x$ as well. This also leads to $w=f(0,0,1) \equiv_{\delta} 0$, which is false, so typ $(\delta, \theta) \notin\{\mathbf{4}, \mathbf{5}\}$.

Finally, assume that $\operatorname{typ}(\delta, \theta) \in\{\mathbf{2}, \mathbf{3}\}$. Continue to assume that $\{0,1\}$ is a $\langle\delta, \theta\rangle$-subtrace for which $w=f(0,0,1) \not \equiv_{\delta} 0$. Let $U$ be a $\langle\delta, \theta\rangle$-minimal set which has a $\langle\delta, \theta\rangle$-trace $N$ containing 0 and 1 . Since $w=f(0,0,1) \equiv_{\theta} f(0,0,0) \equiv_{\delta} 0$, we have $(w, 0) \in \theta-\delta$. According to Lemma 4.7 of [4], this fact together with typ $(\delta, \theta) \in\{\mathbf{2}, \mathbf{3}\}$ jointly imply that it is possible to find an idempotent unary polynomial $e$ such that $e(A)=U$ and $(e(w), e(0))=(e(w), 0) \notin \delta$. This is a key fact which we will refer to at the end of the proof.

It is time to make use of the pentagon shape. Choose a congruence $\lambda$ in the interval $\operatorname{Int}[\sigma, \gamma]$ which is minimal for the property that $\lambda \vee \delta \geq \theta$. Such a congruence exists and is larger than $\sigma$ since $\sigma \vee \delta=\delta \nsupseteq \theta$ while

$$
\gamma \vee \delta \geq \gamma \vee \alpha=\tau \geq \beta \geq \theta
$$

Let $\lambda_{*}$ be a congruence for which $\sigma \leq \lambda_{*} \prec \lambda \leq \gamma$.

Claim 3.3. Let $B$ and $T$ denote the body and tail of $U$, respectively. Let $\Omega$ denote the largest congruence on $\mathbf{A}$ such that $\left.\Omega\right|_{U} \subseteq B^{2} \cup T^{2}$. Then

(1) $B$ is a $\left.\Omega\right|_{U}$-class, and

(2) $\lambda \not \leq \Omega$ and $\lambda_{*} \leq \Omega$.

In particular, $\left.\lambda\right|_{B} \subseteq \lambda_{*}$.

We make two remarks before proving this claim. First, we explain why there is largest congruence satisfying $\left.\Omega\right|_{U} \subseteq B^{2} \cup T^{2}$. Lemma 2.3 of [2] proves that restriction to $U$ is a homomorphism from the lattice Con $(\mathbf{A})$ to the lattice of equivalance relations on $U$. Thus, the join $\Omega$ of all $\Omega^{\prime} \in$ Con $(\mathbf{A})$ which satisfy $\left.\Omega^{\prime}\right|_{U} \subseteq B^{2} \cup T^{2}$ is a largest congruence that satisfies $\left.\Omega\right|_{U} \subseteq B^{2} \cup T^{2}$. Hence $\Omega$ exists and equals the join of all $\Omega^{\prime} \in \operatorname{Con}(\mathbf{A})$ such that $\left.\Omega^{\prime}\right|_{U} \subseteq B^{2} \cup T^{2}$. Our second remark is that, although we know that $\operatorname{typ}(\delta, \theta) \in\{\mathbf{2}, \mathbf{3}\}$, we will arrange the argument so that it works even if $\operatorname{typ}(\delta, \theta)=4$. We will need to know the result in this generality in the proof of Lemma 3.4.

Now, to prove part (1) of the claim, note that the definition $\Omega$ implies that it is the largest congruence on $\mathbf{A}$ such that $B$ is a union of $\left.\Omega\right|_{U^{-}}$ classes. To see that $B$ consists of single $\left.\Omega\right|_{U^{-}}$class, it is enough to show that $B$ is a $\left.\Omega^{\prime}\right|_{U^{-}}$class for some congruence $\Omega^{\prime} \in \operatorname{Con}(\mathbf{A})$ such that $\left.\Omega^{\prime}\right|_{U} \subseteq B^{2} \cup T^{2}$. If the type of $\langle\delta, \theta\rangle$ is $\mathbf{3}$ or $\mathbf{4}$, then by Lemma 4.17 of [2] the congruence $\Omega^{\prime}=\theta$ has this property. If the type of $\langle\delta, \theta\rangle$ is $\mathbf{2}$, then a suitable congruence for $\Omega^{\prime}$ is described in Lemma 4.27(3) of [2]. This proves part (1) of the claim. 
To prove part (2), observe that if $\left.\lambda\right|_{U} \subseteq B^{2} \cup T^{2}$, then the congruences $\left.\lambda\right|_{B},\left.\theta\right|_{B}$ and $\left.\delta\right|_{B}$ would generate a nontrivial pentagon in the lattice $\operatorname{Con}\left(\left.\mathbf{A}\right|_{B}\right)$. But the fact that typ $(\delta, \theta) \in\{\mathbf{2}, \mathbf{3}, \mathbf{4}\}$ implies that $\left.\mathbf{A}\right|_{B}$ has a Mal'tsev polynomial or lattice polynomials (Lemmas 4.17 and 4.20 of [2]), hence Con $\left(\left.\mathbf{A}\right|_{B}\right)$ must be modular. This shows that $\lambda \not \leq \Omega$.

On the other hand, we must have $\left.\lambda_{*}\right|_{U} \subseteq B^{2} \cup T^{2}$. Otherwise there would exist a pair $(r, s) \in \lambda_{*} \cap(B \times T)$. Since $r \in B$ we can find $r^{\prime}$ so that $\left.\left(r, r^{\prime}\right) \in \theta\right|_{U}-\delta$. Depending on the type of $\langle\delta, \theta\rangle,\left.\mathbf{A}\right|_{U}$ either has a pseudo-Mal'tsev polynomial $d(x, y, z)$ or pseudo-meet and pseudo-join polynomials $x \wedge y$ and $x \vee y$. In any case, at least one of the choices $h(x)=d\left(x, r, r^{\prime}\right), x \wedge r^{\prime}$ or $x \vee r^{\prime}$ is a unary polynomial of $\left.\mathbf{A}\right|_{U}$ for which $h(x) \equiv_{\theta} x$ on $U$, and $h(r)=r^{\prime}$. In particular, if we define $s^{\prime}=h(s)$, then we have

- $(r, s) \in \lambda_{*}$,

- $\left(r^{\prime}, s^{\prime}\right)=(h(r), h(s)) \in \lambda_{*}$, and

- $\left.\left(s, s^{\prime}\right) \in \theta\right|_{T} \subseteq \delta$.

These conditions imply that $r \equiv_{\lambda_{*}} s \equiv_{\delta} s^{\prime} \equiv_{\lambda_{*}} r^{\prime}$. From this we get that $\left(r, r^{\prime}\right) \in \theta \wedge\left(\lambda_{*} \vee \delta\right)=\delta$, contradicting our choice of $r^{\prime}$. Hence $\lambda_{*} \leq \Omega$.

Since $\lambda \not \leq \Omega, \lambda_{*} \leq \Omega$, and $\lambda_{*} \prec \lambda$, it follows that $\lambda \wedge \Omega=\lambda_{*}$. From this and the fact that $B$ is an $\left.\Omega\right|_{U}$-class it is clear that $\left.\lambda\right|_{B} \subseteq \lambda \wedge \Omega=\lambda_{*}$. The proof of Claim 3.3 is complete.

Coupling the fact that $\lambda$ is connected modulo $\lambda_{*}$ by $\left\langle\lambda_{*}, \lambda\right\rangle$-traces (Theorem 2.8 of [2]) with the facts that we just established, $\lambda_{*} \subseteq$ $B^{2} \cup T^{2}$ and $\lambda \nsubseteq B^{2} \cup T^{2}$, we see that there is $\left\langle\lambda_{*}, \lambda\right\rangle$-trace $M$ such that $M \cap B \neq \emptyset \neq M \cap T$. Choose $b \in M \cap B$ and $t \in M \cap T$. By Claim 3.3 we must have $(b, t) \notin \lambda_{*}$, so $\{b, t\}$ is a $\left\langle\lambda_{*}, \lambda\right\rangle$-subtrace. From the local equation $f(x, y, y) \approx_{I} x$ we get that $f(b, t, t) \equiv_{\lambda_{*}} b$, so we also have $e f(b, t, t) \equiv_{\lambda_{*}} e(b)=b \in B$. (The polynomial $e$ was fixed in the second paragraph before Claim 3.3.) Recall that $B$ is an $\left.\Omega\right|_{U^{-}}$class, where $\Omega$ is the congruence defined in Claim 3.3. Hence, since $0, b \in B$, we get that ef $(0, t, t) \equiv_{\Omega}$ ef $(b, t, t) \equiv_{\Omega} b$, so ef $(0, t, t) \in B$. Lemma 2.2 of [3] shows that, for any polynomial $p\left(x_{1}, \ldots, x_{n}\right) \in \operatorname{Pol}\left(\left.\mathbf{A}\right|_{U}\right)$, if $t$ is in the tail of $U$ and $p(t, \ldots, t) \in B$, then $p\left(\left.\theta\right|_{U}, \ldots,\left.\theta\right|_{U}\right) \subseteq \delta$. Applying this fact to the binary polynomial ef $(0, x, y)$ we deduce from $e f(0, t, t) \in B$ that $e f\left(0,\left.\theta\right|_{U},\left.\theta\right|_{U}\right) \subseteq \delta$. But this leads to

$$
e(w)=e f(0,0,1) \equiv_{\delta} e f(0,0,0) \equiv_{\delta} e(0)=0,
$$

which contradicts our earlier conclusion that $(e(w), 0) \notin \delta$. This completes the proof. 
Lemma 3.4. Assume that $\mathbf{A}$ is a finite algebra and that $\mathrm{Con}(\mathbf{A})$ has a sublattice isomorphic to the pentagon, labeled as in Lemma 3.2. Let $I=\operatorname{Int}[\sigma, \tau]$ and $J=\operatorname{Int}[\alpha, \beta]$. If $\mathbf{A}$ satisfies both of the local equations

$$
f(x, x, y) \approx_{I} x \text { and } f(x, y, x) \approx_{I} x
$$

then A satisfies the local equation $f(x, y, y) \approx_{J} x$.

Proof. Assume to the contrary that $f(x, x, y) \approx_{I} f(x, y, x) \approx_{I} x$ while $f(x, y, y) \not_{J} x$. Since $f(x, y, y) \approx_{J} x$, there exist a two-element subinterval $K=\operatorname{Int}[\delta, \theta]$ of $J$, and a $\langle\delta, \theta\rangle$-subtrace $\{0,1\}$ such that $w=f(0,1,1) \not \equiv_{\delta} 0$. We shall derive a contradiction to this.

Assume first that typ $(\delta, \theta) \in\{\mathbf{1}, \mathbf{2}\}$. Since $f(x, x, y) \approx_{I} x$ we have

$$
f(0,0,1) \equiv_{\delta} 0 \equiv_{\delta} f(0,0,0) .
$$

By Theorems 5.5 and 5.6 of [2], $\theta$ is abelian over $\delta$ (Definition 3.6 of $[2])$. This allows us to replace the argument in the second position on each side of this equality with 1 and still maintain $a \equiv_{\delta}$-relation. Hence

$$
w=f(0,1,1) \equiv_{\delta} f(0,1,0) .
$$

From $f(x, y, x) \approx_{I} x$ we get $w \equiv_{\delta} 0$, which is false. Hence typ $(\delta, \theta) \notin$ $\{1,2\}$.

Now assume that typ $(\delta, \theta) \in\{\mathbf{4}, \mathbf{5}\}$. Let $U$ be a $\langle\delta, \theta\rangle$-minimal set containing $\{0,1\}$. Since $(w, 0) \in \theta-\delta$ there is a unary polynomial $k$ such that $k(A)=U$ and $(k(w), k(0)) \notin \delta$ (Theorem 2.8(4) of [2]). If $k\left(\left.\theta\right|_{U}\right) \subseteq \delta$, then since $f(x, x, x) \approx_{I} x$ we get that

$$
k f(0,0,0) \equiv_{\delta} k(0) \equiv_{\delta} k(1) \equiv_{\delta} k f(1,1,1) .
$$

In the $\langle\delta, \theta\rangle$-preorder of the $\theta$-class of 0 the element $k f(0,1,1)$ is between the comparable elements $k f(0,0,0)$ and $k f(1,1,1)$. The latter two elements are $\delta$-related, so $k(w)=k f(0,1,1) \equiv_{\delta} k f(0,0,0) \equiv_{\delta} k(0)$, contrary to the choice of $k$. Consequently $\left.k\right|_{U}$ is a permutation. Since $k$ preserves the $\langle\delta, \theta\rangle$-preorder we must have $k(x) \equiv_{\delta} x$ for all $x$ in the body of $U$. Thus, since $k(w) \not_{\delta} k(0) \equiv_{\delta} 0 \not \equiv_{\delta} 1$, we get $1 \equiv_{\delta} k(w) \equiv_{\delta}$ $k f(0,1,1)$. Together with

$$
k f(x, y, x) \equiv_{\delta} k(x) \equiv_{\delta} x
$$

on $N$, and $k f(x, x, y) \equiv_{\delta} x$ on $N$, it is not hard to show that $k f(0, x, y)$ and $k f(x, y, 1)$ are meet and join modulo $\delta$ on the body of $U$ with respect to the order $0<1$. The conclusions we draw are that typ $(\delta, \theta) \neq$ $\mathbf{5}$, and that if $\operatorname{typ}(\delta, \theta)=\mathbf{4}$ then $\left.k\right|_{U}$ is a permutation. In this situation, let $e$ be an idempotent iterate of $k$. The polynomial $e$ has the properties that $e(A)=U$ and $(e(w), e(0))=(e(w), 0) \in \theta-\delta$.

In the remaining case, when typ $(\delta, \theta)=3$, Lemma 4.7 of [4] guarantees the existence of an idempotent unary polynomial $e$ with the same 
properties: $e(A)=U$ and $(e(w), e(0))=(e(w), 0) \in \theta-\delta$. We have now shown that typ $(\delta, \theta) \in\{\mathbf{3}, \mathbf{4}\}$, and that for either type we have a special polynomial $e$.

Choose a congruence $\lambda$ in the interval $\operatorname{Int}[\sigma, \gamma]$ which is minimal for the property that $\lambda \vee \delta \geq \theta$ and choose $\lambda_{*}$ such that $\sigma \leq \lambda_{*} \prec \lambda$. As in the proof of Claim 3.3 of Lemma 3.2, if $B$ and $T$ denote the body and tail of $U$, then $\left.\lambda\right|_{U} \nsubseteq B^{2} \cup T^{2}$ while $\left.\lambda_{*}\right|_{U} \subseteq B^{2} \cup T^{2}$.

Claim 3.5. There exist $\left\langle\lambda_{*}, \lambda\right\rangle$-subtraces $\{0, u\}$ and $\{v, 1\}$ such that $(u, v) \in \delta$.

As argued after the proof of Claim 3.3 of Lemma 3.2, there is a $\left\langle\lambda_{*}, \lambda\right\rangle$-trace $M$ such that $M \cap B \neq \emptyset \neq M \cap T$. Choose $r \in M \cap B$ and $s \in M \cap T$. Since $(r, s) \in(B \times T)$ we must have $(r, s) \notin \lambda_{*}$. Therefore $\{r, s\}$ is a $\left\langle\lambda_{*}, \lambda\right\rangle$-subtrace. Since $r \in B$ we can find $r^{\prime}$ so that $\left.\left(r, r^{\prime}\right) \in \theta\right|_{U}-\delta$. As argued in the proof of Claim 3.3, there is a unary polynomial $h$ of $\left.\mathbf{A}\right|_{U}$ for which $h(x) \equiv_{\theta} x$ on $U$, and $h(r)=r^{\prime}$. Define $s^{\prime}=h(s)$. We have

- $\left\{r, r^{\prime}\right\}$ is a $\langle\delta, \theta\rangle$-subtrace in $U$,

- $\{r, s\}$ is a $\left\langle\lambda_{*}, \lambda\right\rangle$-subtrace,

- $\left.\left(s, s^{\prime}\right) \in \theta\right|_{T} \subseteq \delta$, and

- $\left(r^{\prime}, s^{\prime}\right)=(h(r), h(s)) \in \lambda \cap(B \times T)$.

Since $\left\{r^{\prime}, s^{\prime}\right\}$ is a polynomial image of the $\left\langle\lambda_{*}, \lambda\right\rangle$-subtrace $\{r, s\}$ and $\left(r^{\prime}, s^{\prime}\right) \notin \lambda_{*}$ it follows that $\left\{r^{\prime}, s^{\prime}\right\}$ is also a $\left\langle\lambda_{*}, \lambda\right\rangle$-subtrace.

Since typ $(\delta, \theta) \in\{\mathbf{3}, \boldsymbol{4}\}$ the set $\{0,1\}$ is the unique $\langle\delta, \theta\rangle$-subtrace in $U$, so $\left\{r, r^{\prime}\right\}=\{0,1\}$. If $r=0$ and $r^{\prime}=1$ then we take $u$ and $v$ to be $s$ and $s^{\prime}$, while if $r=1$ and $r^{\prime}=0$ then we take $u$ and $v$ to be $s^{\prime}$ and $s$. All the assertions of the claim are proved.

Consider the following elements of $U$.

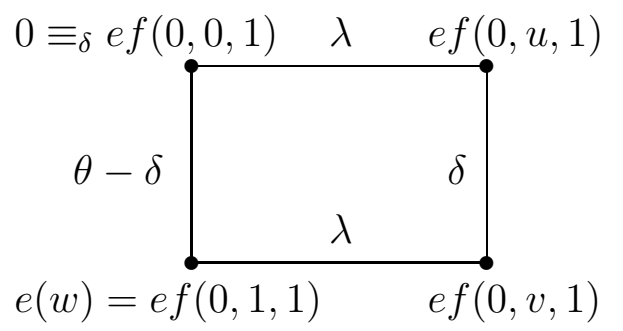

In this graph, an edge between a pair of elements is labeled by a relation only when we know those elements to be so related. All relations indicated follow from the previously determined facts that $(e(w), e(0))=(e(w), 0) \in \theta-\delta$ and $0 \equiv_{\lambda} u \equiv_{\delta} v \equiv_{\lambda} 1$. 
Claim 3.6. The elements of the previous figure lie in the body of $U$

It is clear that $\operatorname{ef}(0,0,1) \equiv_{\delta} 0 \in B$ and $\operatorname{ef}(0,1,1)=e(w) \in B$. Since $\{1, v\}$ is a $\left\langle\lambda_{*}, \lambda\right\rangle$-subtrace and $f(x, y, x) \approx_{I} x$, we get that ef $(1, v, 1) \equiv_{\lambda_{*}} e(1)=1$. But since $\lambda_{*} \vee \delta \nsupseteq \theta$ and $B=\{0,1\}$, we must have $\left.\lambda_{*}\right|_{B}=0_{B}$. Hence ef $(0, v, 1) \equiv_{\theta}$ ef $(1, v, 1)=1 \in B$, which implies that ef $(0, v, 1) \in B$. Similarly ef $(0, u, 1) \equiv_{\theta}$ ef $(0, u, 0)=0 \in B$. This completes the proof of the claim.

Since $B^{2}=\{0,1\}^{2} \subseteq \theta$, this claim shows that all of the elements in the previous figure are $\left.\theta\right|_{U}$-related. Therefore, since $\lambda \wedge \theta \leq \delta$,

$$
e(w)=e f(0,1,1) \equiv_{\delta} \operatorname{ef}(0, v, 1) \equiv_{\delta} \operatorname{ef}(0, u, 1) \equiv_{\delta} \text { ef }(0,0,1) \equiv_{\delta} 0,
$$

which contradicts the fact that $(e(w), 0) \notin \delta$. The proof is finished.

Theorem 3.7. Let $\mathcal{V}$ be a locally finite variety. The following conditions are equivalent.

(1) $\mathcal{V}$ satisfies a nontrivial congruence identity.

$(2) \mathcal{V}$ satisfies an idempotent Mal'tsev condition which fails in the variety of semilattices.

(3) typ $\{\mathcal{V}\} \subseteq\{\mathbf{2}, \mathbf{3}, \mathbf{4}\}$

(4) $\mathcal{V} \models_{c o n} \alpha \cap(\beta \circ \gamma) \subseteq(\gamma \vee(\alpha \wedge \beta)) \circ(\beta \vee(\alpha \wedge \gamma))$

(5) There exists $k<\omega$ and 3 -ary terms $d_{0}, \ldots, d_{2 k+1}, e_{0}, \ldots, e_{2 k+1}, p$ such that $\mathcal{V}$ satisfies the following equations:

(i) $d_{0}(x, y, z) \approx p(x, y, z) \approx e_{0}(x, y, z)$;

(ii) $d_{i}(x, y, y) \approx d_{i+1}(x, y, y)$ and $e_{i}(x, x, y) \approx e_{i+1}(x, x, y)$ for even $i$;

(iii) $d_{i}(x, x, y) \approx d_{i+1}(x, x, y), d_{i}(x, y, x) \approx d_{i+1}(x, y, x)$, $e_{i}(x, y, y) \approx e_{i+1}(x, y, y)$ and $e_{i}(x, y, x) \approx e_{i+1}(x, y, x)$ for odd $i$

(iv) $d_{2 k+1}(x, y, z) \approx x$ and $e_{2 k+1}(x, y, z) \approx z$.

(6) There exists $\ell<\omega$ such that the lattice $\mathbf{N}_{\ell}$ cannot be embedded into $\operatorname{Con}(\mathbf{A})$ for any finite $\mathbf{A} \in \mathcal{V}$.

Proof. The implication $(6) \Rightarrow(1)$ follows from Theorem 2.1. The implications $(1) \Rightarrow(2) \Leftrightarrow(3) \Leftrightarrow(4) \Leftrightarrow(5)$ are from Theorems 9.8 and 9.18 of [2]. (Our notation in (5) differs slightly from that of [2].) The rest of the proof will be devoted to establishing the implication $(5) \Rightarrow(6)$.

Fix a $k$ for which (5) holds. Assume that $\mathbf{N}_{2 k}$ is a sublattice of the congruence lattice of some finite $\mathbf{A} \in \mathcal{V}$. Label the elements of the sublattice as in the following figure. 


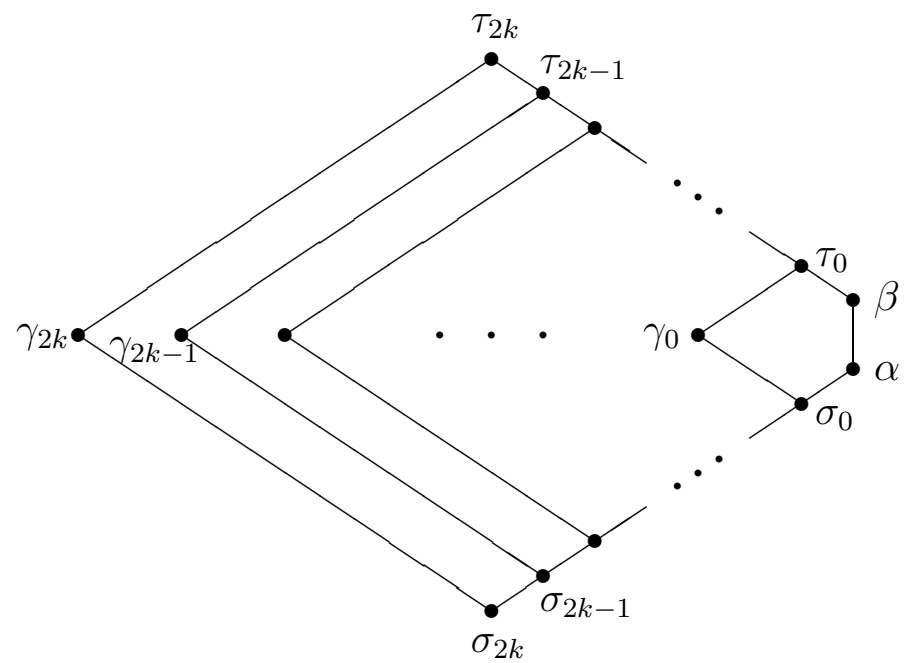

By (5)(ii) and (5)(iv), the algebra A satisfies the equations

$$
d_{2 k}(x, y, y) \approx d_{2 k+1}(x, y, y) \text { and } d_{2 k+1}(x, y, z) \approx x .
$$

In particular, this implies that $d_{2 k}(x, y, y) \approx_{I} x$ for $I=\operatorname{Int}\left[\sigma_{2 k}, \tau_{2 k}\right]$.

Assume that for some $i$ satisfying $0 \leq i<k$ we have shown that $d_{2 k-2 i}(x, y, y) \approx_{I} x$ holds for $I=\operatorname{Int}\left[\sigma_{2 k-2 i}, \tau_{2 k-2 i}\right]$. By Lemma 3.2 we have that

$$
d_{2 k-2 i}(x, x, y) \approx_{J} x \text { and } d_{2 k-2 i}(x, y, x) \approx_{J} x
$$

hold for $J=\operatorname{Int}\left[\sigma_{2 k-2 i-1}, \tau_{2 k-2 i-1}\right]$. By (5)(iii) we have that $\mathbf{A}$ satisfies $d_{2 k-2 i-1}(x, x, y) \approx d_{2 k-2 i}(x, x, y)$ and $d_{2 k-2 i-1}(x, y, x) \approx d_{2 k-2 i}(x, y, x)$, SO

$$
d_{2 k-2 i-1}(x, x, y) \approx_{J} x \text { and } d_{2 k-2 i-1}(x, y, x) \approx_{J} x .
$$

This establishes the hypotheses of Lemma 3.4 for $f=d_{2 k-2 i-1}$. The conclusion of that lemma is that the local equation $d_{2 k-2 i-1}(x, y, y) \approx_{K}$ $x$ holds for $K=\operatorname{Int}\left[\sigma_{2 k-2 i-2}, \tau_{2 k-2 i-2}\right]$. Now, by (5)(ii), A satisfies $d_{2 k-2 i-2}(x, y, y) \approx d_{2 k-2 i-1}(x, y, y)$, hence $d_{2 k-2 i-2}(x, y, y) \approx_{K} x$. To summarize, we have shown that

(a) $d_{2 k}(x, y, y) \approx_{I} x$ holds for $I=\operatorname{Int}\left[\sigma_{2 k}, \tau_{2 k}\right]$, and

(b) if $d_{2 k-2 i}(x, y, y) \approx_{I} x$ holds for $I=\operatorname{Int}\left[\sigma_{2 k-2 i}, \tau_{2 k-2 i}\right]$, then $d_{2 k-2(i+1)}(x, y, y) \approx_{K} x$ holds for $K=\operatorname{Int}\left[\sigma_{2 k-2(i+1)}, \tau_{2 k-2(i+1)}\right]$.

By induction, $d_{0}(x, y, y) \approx_{L} x$ holds for $L=\operatorname{Int}\left[\sigma_{0}, \tau_{0}\right]$. The same argument works for the $e_{j}$ 's in place of the $d_{j}$ 's. (One should reverse the order of the variables in the $e_{j}$ 's in order to use Lemmas 3.2 and 3.4 in the form in which they are stated.) Altogether this proves that $d_{0}(x, y, y) \approx_{L} x$ and $e_{0}(y, y, x) \approx_{L} x$ for $L=\operatorname{Int}\left[\sigma_{0}, \tau_{0}\right]$. But (5)(i) states that

$$
d_{0}(x, y, z) \approx p(x, y, z) \approx e_{0}(x, y, z)
$$


so the term $p(x, y, z)$ in condition (5) is a term satisfying $p(x, y, y) \approx_{L}$ $x$ and $p(y, y, x) \approx_{L} x$. Applying Lemma 3.2 to $p(x, y, y) \approx_{L} x$ we find that $x \approx_{M} p(x, x, y)$ for $M=\operatorname{Int}[\alpha, \beta]$, while $p(y, y, x) \approx_{L} x$ trivially implies $p(x, x, y) \approx_{M} y$. Hence $x \approx_{M} y$. But the definition of a subtrace implies that the local equation $x \approx_{M} y$ never holds when $M$ is a nontrivial interval. This contradiction shows that $\mathbf{N}_{2 k}$ is not a sublattice of $\operatorname{Con}(\mathbf{A})$ for any finite $\mathbf{A} \in \mathcal{V}$.

\section{REFERENCES}

[1] R. Freese and J. B. Nation, Congruence lattices of semilattices, Pacific J. Math. 49 (1973), 51-58.

[2] D. Hobby and R. McKenzie, The Structure of Finite Algebras, Contemporary Mathematics v. 76, American Mathematical Society, 1988.

[3] K. A. Kearnes and E. W. Kiss, Modularity prevents tails, to appear in Proc. Amer. Math. Soc.

[4] K. A. Kearnes, E. W. Kiss and M. A. Valeriote, A geometric consequence of residual smallness, to appear in J. Pure and Appl. Logic.

[5] R. McKenzie, Equational bases and nonmodular lattice varieties, Trans. Amer. Math. Soc. 174 (1972), 1-43.

[6] A. Pixley, Local Mal'cev conditions, Canad. Math. Bull. 15 (1972), 559-568.

[7] R. Wille, Kongruenzklassengeometrien, Lecture Notes in Mathematics 113 (Springer-Verlag, Berlin, 1970).

(Keith Kearnes) Department of Mathematics, University of Louisville, LOUISVILle, KY 40292, USA

E-mail address: kearnes@louisville.edu 